\title{
SYSTEMS-CONJUGATE AND FOCAL POINTS OF FOURTH ORDER NONSELFADJOINT DIFFERENTIAL EQUATIONS
}

\author{
BY \\ SUI-SUN CHENG
}

\begin{abstract}
Systems-conjugate points have been studied by John Barrett in relation to selfadjoint fourth order differential equations of the form $\left(p_{2} u^{\prime \prime}\right)^{\prime \prime}+$ $p_{0} u=0$. This paper extends his results to the general nonselfadjoint fourth order differential equation via a system of second order equations.
\end{abstract}

1. Introduction. With reference to the following system of second order differential equations

$$
\left(\begin{array}{l}
x \\
y
\end{array}\right)^{\prime \prime}+\left(\begin{array}{ll}
a(t) & b(t) \\
c(t) & d(t)
\end{array}\right)\left(\begin{array}{l}
x \\
y
\end{array}\right)=0
$$

where $a(t), d(t) \geqslant 0, b(t), c(t)>0$ are continuous on $[\alpha, \infty)$, the systems-conjugate point $\hat{\eta}_{1}(\alpha)$ [systems-focal point $\hat{\mu}_{1}(\alpha)$ ] is defined as the smallest $\beta \in$ $(\alpha, \infty)$ such that $(x, y)(\alpha)=0=(x, y)(\beta)\left[(x, y)(\alpha)=0=(x, y)^{\prime}(\beta)\right]$ is satisfied by a nontrivial solution of (1).

It is easy to see that the following special selfadjoint fourth order equation

$$
l[u] \equiv\left(p_{2}(t) u^{\prime \prime}\right)^{\prime \prime}+p_{0}(t) u=0
$$

can be represented in the form (1) with $x(t)=u(t), b(t)=-1 / p_{2}(t)>0, p_{0}(t)$ $=c(t)$ and $a(t)=d(t) \equiv 0$. Whyburn [1] showed that the general real selfadjoint fourth order equation

$$
l[u] \equiv\left(p_{2}(t) u^{\prime \prime}\right)^{\prime \prime}-\left(p_{1}(t) u^{\prime}\right)^{\prime}+p_{0}(t) u=0
$$

can be written in the form (1) with $b(t)=-1 / p_{2}(t)>0$ and $a(t) \equiv d(t)$. Later Kreith [2] also showed the general real linear fourth order equation

$$
l[u] \equiv\left(p_{2}(t) u^{\prime \prime}-q_{2}(t) u^{\prime}\right)^{\prime \prime}-\left(p_{1}(t) u^{\prime}-q_{1}(t) u\right)^{\prime}+p_{0}(t) u=0
$$

can be reduced to the form (1), with the nonselfadjointness reflected by the inequality of $a(t)$ and $d(t)$.

Presented to the Society, April 12, 1975; received by the editors April 24, 1975.

AMS (MOS) subject classifications (1970). Primary 34C10.

Key words and phrases. System of second order differential equations, systems-conjugate point, systems-focal point, $\theta$-principal solutions, reciprocal system, comparison theorems. 
Henceforth, it is assumed that (4) is represented by (1) and $a(t) \geqslant 0$, $d(t) \geqslant 0, b(t)>0, c(t)>0$ are continuous on $[\alpha, \infty)$. This paper is then concerned primarily with the existence of $\hat{\eta}_{1}(\alpha)$ and $\hat{\mu}_{1}(\alpha)$ which Barrett [3] originally defined as the systems-conjugate and systems-focal points of $\alpha$ with respect to (2). Actually systems of the form (2) had been considered earlier by Bliss and Schoenberg [4], Hartman and Wintner [5] and others in connection with the calculus of variations which naturally requires the coefficient matrix in (2) to be symmetric. Such an assumption, however, is not required here.

Simple physical interpretation of the second order equation

$$
u^{\prime \prime}+q(t) u=0, \quad u(\alpha)=0
$$

motivates a variety of well-known oscillation and disconjugacy criteria. This paper follows a similar pattern in that (1) allows a simple dynamical interpretation in terms of a particle of unit mass in a force field $F=(-a x-b y,-c x-d y)$. In this context $\hat{\eta}_{1}(\alpha)\left[\hat{\mu}_{1}(\alpha)\right]$ is the minimal time for the particle, being fired from the origin, to return to the origin [to stop]. Since this force field is purely attractive in the first and third quadrants, one might conjecture the following:

(i) $\hat{\eta}_{1}(\alpha), \hat{\mu}_{1}(\alpha)$, if they exist, will be realized by trajectories contained entirely in the first or third quadrant on $\left(\alpha, \hat{\eta}_{1}(\alpha)\right),\left(\alpha, \hat{\mu}_{1}(\alpha)\right)$ respectively.

(ii) $\hat{\eta}_{1}(\alpha)$ exists iff $\hat{\mu}_{1}(\alpha)$ exists. Moreover, $\hat{\mu}_{1}(\alpha)<\hat{\eta}_{1}(\alpha)$.

(iii) A force field for which $\hat{\eta}_{1}(\alpha)$ exists implies the existence of smaller conjugate point for any stronger force field.

(iv) If the attractive force is "strong" enough, then $\hat{\eta}_{1}(\alpha)$ and $\hat{\mu}_{1}(\alpha)$ exist.

In $\S \S 2-5$, the above statements will be made precise and proved. Disconjugacy criteria are established in $\S 6$, while application to fourth order equations are presented in $\$ 7$.

2. Elementary properties of solutions. We begin by establishing a series of lemmas for solutions of (1).

2.1. LEMMA. Let $(\bar{x}, \bar{y})$ be a solution of (1). If $\bar{x}(t)>0$ and $\bar{y}(t)>0$ on $(\alpha, \infty)$, then

(i) $\bar{x}^{\prime \prime}<0$ and $\bar{y}^{\prime \prime}<0$ on $(\alpha, \infty)$.

(ii) $\bar{x}^{\prime}>0$ and $\bar{y}^{\prime}>0$ on $(\alpha, \infty)$.

Proof. (i) follows directly from (1).

(ii) Assume to the contrary that there exists $\beta>\alpha$ so that $\bar{x}^{\prime}(\beta)<0$. Then an iterated integration of $\vec{x}^{\prime \prime}<0$, yields

$$
\bar{x}(t)=\bar{x}(\beta)+\bar{x}^{\prime}(\beta)(t-\beta)+\int_{\beta}^{t} \int_{\beta}^{\xi} \bar{x}^{\prime \prime}(\xi) d \xi d t .
$$

Now as $t \rightarrow \infty$, the right side will eventually be negative contradicting the assumption $\bar{x}(t)>0 . \bar{y}^{\prime}>0$ can be proved similarly. Q.E.D. 
2.2. Lemma. Let $(\bar{x}, \bar{y})$ be a solution of (1). If $\bar{x}(t)>0$ and $\bar{y}(t)>0$ on $(\alpha, \beta)$, then $u^{\prime \prime}+a(t) u=0$ and $v^{\prime \prime}+d(t) v=0$ are both disconjugate on $(\alpha, \beta)$.

Proof. Assume to the contrary that $\bar{u}$ is a solution of $u^{\prime \prime}+a(t) u=0$ such that $\bar{u}(\gamma)=\bar{u}(\delta)=0$ and $\bar{u}(t)>0$ on $(\gamma, \delta)$ where $\alpha \leqslant \gamma<\delta<\beta$. From (1) we have

$$
\bar{x}^{\prime \prime}+a \bar{x}=-b \bar{y}<0 \quad \text { on }(\alpha, \beta) .
$$

Hence $\left[\vec{x} \vec{u}^{\prime}-\bar{u} \bar{x}^{\prime}\right]=\bar{x} \vec{u}^{\prime \prime}-\bar{u} \vec{x}^{\prime \prime}=-\bar{u}(\bar{x}+a \bar{x})>0$ on $(\gamma, \delta)$ which yields the following contradiction.

$$
0 \geqslant \bar{x}(\delta) \bar{u}^{\prime}(\delta)-\bar{x}(\gamma) \bar{u}^{\prime}(\gamma)=\bar{x} \bar{u}^{\prime}-\left.\bar{u} \bar{x}^{\prime}\right|_{\gamma} ^{\delta}>0 .
$$

The disconjugacy of $v^{\prime \prime}+d v=0$ is similarly proved. Q.E.D.

2.3.1. Lemma. If there is a solution $(\bar{x}, \bar{y})$ of (1) such that $(\bar{x}, \bar{y})(\alpha)=$ 0 and $\bar{x}(t)>0, \bar{y}(t)>0$ on $(\alpha, \beta)$, then for all solutions $(x, y)$ of $(1)$ such that $(x, y)(\alpha)=0, x^{\prime}(\alpha) \leqslant 0$ and $y^{\prime}(\alpha) \geqslant 0$ (but not both zero) we have $x(t)<0$, $y(t)>0$ on $(\alpha, \beta]$.

Proof. Assume to the contrary there is a solution of $(1)$ with $(x, y)(\alpha)$ $=0, x^{\prime}(\alpha) \leqslant 0, y^{\prime}(\alpha) \geqslant 0$. We can assume then $x(\alpha)=x(\delta)=0$ but $x(t)<0$, $y(t)>0$ on $(\alpha, \delta)$ where $\delta<\beta$. Then $\bar{x}^{\prime \prime}+a \bar{x}=-b \bar{y}<0$ implies $\left[\bar{x} x^{\prime}-x \bar{x}^{\prime}\right]^{\prime}$ $=\bar{x} x^{\prime \prime}-x \bar{x}^{\prime \prime}=-\bar{x} b y-x\left(\bar{x}^{\prime \prime}+a \bar{x}\right) \leqslant 0$ on $(\alpha, \delta)$. However $0 \leqslant \bar{x}(\delta) x^{\prime}(\delta)=$ $\left[\bar{x} x^{\prime}-x \bar{x}^{\prime}\right]_{\alpha}^{\delta}<0$ is a contradiction. Q.E.D.

These lemmas have simple physical interpretations. In particular, Lemma 2.3.1 asserts that no trajectory can remain in the first quadrant longer than any trajectory that remains in the second quadrant.

Arguments similar to those in the proof of Lemma 2.3.1 establish the following

2.3.2. Lemma. If there exists a solution $(\bar{x}, \bar{y})$ of $(1)$ such that $(\bar{x}, \bar{y})(\alpha)$ $=0$ and $\bar{x}^{\prime}(t)>0, \bar{y}^{\prime}(t)>0$ on $(\alpha, \beta)$, then for all solutions $(x, y)$ of $(1)$ such that $(x, y)(\alpha)=0$ and $x^{\prime}(\alpha) \leqslant 0, y^{\prime}(\alpha) \geqslant 0$, we have $x^{\prime}(t)<0, y^{\prime}(t)>0$ on $(\alpha, \beta]$.

3. $\theta$-principal solutions. Physical considerations suggest that $\hat{\eta}_{1}(\alpha)$ and $\hat{\mu}_{1}(\alpha)$ will be realized by trajectories completely contained in the first quadrant. For this reason, we begin by restricting our attention to trajectories which lie in the first quadrant for sufficiently small $t>\alpha$.

3.1. Definition. A solution $(x, y)$ of $(1)$ is a principal solution if $(x, y)(\alpha)=0$ and $(x, y)^{\prime}(\alpha)=(\cos \theta, \sin \theta)$ where $\theta \in(0, \pi / 2)$. 
Note that a principal solution is characterized by a single parameter $\theta$ so that $\left(x^{\prime 2}+y^{\prime 2}\right)(\alpha)=1$. In what follows a principal solution (which is only defined for $\theta \in(0, \pi / 2))$ is denoted by $(x, y)_{\theta}$ and its components by $x_{\theta}, y_{\theta}$. Some immediate properties of the principal solutions are as follows.

3.2. Lemma. There exists a principal solution $(x, y)_{\varphi}$ such that $x_{\varphi}(t)>$ $0, y_{\varphi}(t)>0$ on some $(\alpha, \beta)$ but $x_{\varphi}(\beta)=0$.

Proof. Consider the solution $(\bar{x}, \bar{y})$ determined by $(\bar{x}, \bar{y})(\alpha)=0$ and $(\bar{x}, \bar{y})^{\prime}(\alpha)=(0,1)$. Now $\bar{y}^{\prime}(\alpha)=1$ implies $\bar{y}(t)>0$ on some $(\alpha, \delta)$. Also from (1), $\bar{x}(t)<0$ on some $(\alpha, \gamma)$ otherwise, $\bar{x}(t) \geqslant 0$ on $(\alpha, \gamma)$ implies $\bar{x}^{\prime \prime}(t)=-a \bar{x}$ $-b \bar{y}<0$ on $(\alpha, \min (\delta, \gamma))$. Hence $\bar{x}(t)<0$ on $(\alpha, \min (\delta, \gamma))$ by interated integration which is a contradiction. Now by continuous dependence of the solutions on the parameter $\theta$, for $\varphi$ sufficiently close to $\pi / 2,(x, y)_{\varphi}$ will satisfy $x_{\varphi}(t)>$ $0, y_{\varphi}(t)>0$ on some $(\alpha, \beta)$ and $x_{\varphi}(\beta)=0$. Q.E.D.

Hence Lemma 3.2 guarantees the existence of principal solutions that exit from the first quadrant into the second or possibly into the third quadrant.

3.3. LEMMA. There exists a principal solution $(x, y)_{\psi}$ such that $x_{\psi}^{\prime}(t)>$ 0 and $y_{\psi}^{\prime}(t)>0$ on some $(\alpha, \xi)$ but $x_{\psi}^{\prime}(\xi)=0$.

PROOF. This is a consequence of Lemma 3.2 and Rolle's theorem.

Actually once a principal solution is known to leave the first quadrant, the same is true of an infinite class of solutions. But before specifying this class, some definitions are necessary.

3.4.1. Definition. I II is the set of principal solutions $(x, y)_{\theta}$ that satisfy $x_{\theta}(t)>0, y_{\theta}(t)>0$ on some $(\alpha, \beta(\theta))$ but $x_{\theta}(\beta(\theta))=0$ (i.e., a first zero $\beta(\theta)$ exists for $x_{\theta}$ so that $y_{\theta}(t)>0$ on $(\alpha, \beta(\theta))$.

3.4.2. Definition. I II' is the set of principal solutions $(x, y)_{\theta}$ that satisfy $x_{\theta}^{\prime}(t)>0, y_{\theta}^{\prime}(t)>0$ on some $(\alpha, \xi(\theta))$ but $x_{\theta}^{\prime}(\xi(\theta))=0$.

By exchanging the role of $x$ and $y$ in Lemmas 3.2 and 3.3, it is also possible to make the following definitions.

3.4.3. DEFInition. I IV is the set of principal solutions $(x, y)_{\theta}$ that satisfy $x_{\theta}(t)>0, y_{\theta}(t)>0$ on some $(\alpha, \beta(\theta))$ but $y_{\theta}(\beta(t))=0$.

3.4.4. Definition. I IV' is the set of principal solutions $(x, y)_{\theta}$ that satisfy $x_{\theta}^{\prime}(t)>0, y_{\theta}^{\prime}(t)>0$ on some $(\alpha, \xi(\theta))$ but $y_{\theta}^{\prime}(\xi(\theta))=0$.

The next theorem is an important result which establishes the monotonicity of $\beta(\theta)$.

3.5.1. THEOREM. If $(x, y)_{\varphi} \in \mathrm{I}$ II, then for all $\theta>\varphi,(x, y)_{\theta} \in \mathrm{I}$ II. Moreover, if $\beta(\theta), \beta(\varphi)$ are the first zeros of $x_{\theta}, x_{\varphi}$ respectively, then $\beta(\theta)<\beta(\varphi)$.

PRoof. Suppose $\beta(\varphi)$ is the first zero of $x_{\varphi}$ so that $y_{\varphi}(t)>0$ on $(\alpha, \beta(\varphi))$, 
then $(x, y)=(x, y)_{\theta}-(x, y)_{\varphi}$ is a solution of (1) such that $(x, y)(\alpha)=0$ and $x^{\prime}(\alpha)<0, y^{\prime}(\alpha)>0$. Hence by Lemma $2.3, x(t)<0$ and $y(t)>0$ on $(\alpha, \beta(\varphi)]$. Now if $x_{\theta}(t)>0$ on $(\alpha, \beta(\varphi)]$, then $x(\beta(\varphi))=x_{\theta}(\beta(\varphi))>0$ is a contradiction. Thus $x_{\theta}$ has a first zero $\beta(\theta)<\beta(\varphi)$ and moreover $(x, y)_{\theta} \in$ I II $\cup$ I IV. To complete the proof, it must be shown that for all $\theta>\varphi,(y, z)_{\theta} \notin$ I IV. Assume to the contrary that for some $\psi>\varphi,(x, y)_{\psi} \in \mathrm{I}$ IV. Let $A=\left\{\theta \in[\varphi, \pi / 2) \mid(x, y)_{\theta}\right.$ $\in$ I II $\}, B=\left\{\theta \in[\varphi, \pi / 2) \mid(x, y)_{\theta} \in \mathrm{I}\right.$ IV $\}$. Then $A \cup B=[\varphi, \pi / 2) . \quad A \neq \varnothing$, $B \neq \varnothing$. By continuous dependence of the principal solutions of the parameter $\theta, A$ and $B$ are also open. Hence $\{A, B\}$ is a disconnection of the connected interval $[\varphi, \pi / 2)$ which is the desired contradiction. Q.E.D.

Similar techniques establish the following,

3.5.2. THEOREM. If $(x, y)_{\varphi} \in \mathrm{I} I \mathrm{IV}$, then for all $\theta<\varphi,(x, y)_{\theta} \in \mathrm{I}$ IV. Moreover, if $\beta(\varphi), \beta(\theta)$ are the first zeros of $y_{\varphi}, y_{\theta}$ respectively, then $\beta(\theta)<\beta(\varphi)$.

3.5.3. THEOREM. If $(x, y)_{\varphi} \in \mathrm{I} \mathrm{II}^{\prime}$, then for all $\theta<\varphi,(x, y)_{\theta} \in \mathrm{I} \mathrm{II}^{\prime}$. Moreover, if $\beta(\varphi), \beta(\theta)$ are the first zeros of $x_{\varphi}^{\prime}$, $x_{\theta}^{\prime}$ respectively, then $\beta(\theta)<\beta(\varphi)$.

3.5.4. THEOREM. If $(x, y)_{\varphi} \in \mathrm{I} \mathrm{IV}^{\prime}$, then for all $\theta<\varphi,(x, y)_{\theta} \in \mathrm{I} \mathrm{IV}^{\prime}$. Moreover, if $\beta(\varphi), \beta(\theta)$ are the first zeros of $y_{\varphi}^{\prime}, y_{\theta}^{\prime}$ respectively, then $\beta(\theta)<\beta(\varphi)$.

3.6.1. COROLLARY. If there exists a principal solution $(x, y)_{\phi} \in \mathrm{I}$ II $\cap$ I IV, then for all $\theta \in(0, \pi / 2),(x, y)_{\theta} \in$ I II U I IV.

3.6.2. COROLlaRY. If there exists a principal solution $(x, y)_{\phi} \in \mathrm{III}^{\prime} \cap$ I IV', then for all $\theta \in(0, \pi / 2),(x, y)_{\theta} \in \mathrm{IIII}^{\prime} \cup \mathrm{I} \mathrm{IV}^{\prime}$.

4. Relations between systems-conjugacy and focal conditions. Before proving the major theorems, systems-conjugate and focal points for $\theta$-principal solutions will be introduced.

4.1.1. Definition. $\bar{\eta}_{1}(\alpha)$ is the smallest $\beta \in(\alpha, \infty)$ such that $(x, y)(\alpha)$ $=0=(x, y)(\beta)$ and $x(t)>0, y(t)>0$ on $(\alpha, \beta)$ are satisfied by a nontrivial solution of (1).

4.1.2. Definition. $\bar{\mu}_{1}(\alpha)$ is the smallest $\beta \in(\alpha, \infty)$ such that $(x, y)(\alpha)$ $=0=(x, y)^{\prime}(\beta)$ and $x^{\prime}(t)>0, y^{\prime}(t)>0$ on $(\alpha, \beta)$ are satisfied by a nontrivial solution of (1).

Note that $\bar{\eta}_{1}(\alpha), \bar{\mu}_{1}(\alpha)$ are necessarily attained by principal solutions. Moreover if $\bar{\eta}_{1}(\alpha)$ exists, then the principal solution for which $\bar{\eta}_{1}(\alpha)$ is attained belongs to I II $\cap$ I IV. Conversely, if there is a principal solution which belongs to I II $\cap$ I IV, then by Theorem 3.5.1, it is the essentially unique solution for which $\bar{\eta}_{1}(\alpha)$ is attained.

4.2.1. THEOREM. $\bar{\eta}_{1}(\alpha)$ exists iff for all $\theta \in(0, \pi / 2),(x, y)_{\theta} \in$ I II U I IV. 
ProOF. By the above remark, $\bar{\eta}_{1}(\alpha)$ exists if and only if I II $\cap$ I IV $\neq \varnothing$. Now if $(x, y)_{\varphi} \in$ I II $\cap$ I IV, then for all $\theta \in(0, \pi / 2),(x, y)_{\theta} \in$ I II U I IV by Corollary 3.6.1. To show the "if" part, assume I II $\cap$ I IV $=\varnothing$. Let $A=\left\{\theta \in(0, \pi / 2) \mid(x, y)_{\theta} \in \mathrm{I}\right.$ II $\}, B=\left\{\theta \in(0, \pi / 2) \mid(x, y)_{\theta} \in \mathrm{I} \mathrm{IV}\right\}$. By Lemma 3.2, $A, B \neq \varnothing$, while the assumption $(x, y)_{\theta} \in$ I II $\cup$ I IV for all $\theta \in(0, \pi / 2)$ implies $A \cup B=(0, \pi / 2)$. Also $A$ and $B$ are open by continuous dependence of $(x, y)_{\theta}$ on $\theta$. Hence $\{A, B\}$ is a disconnection of the connected interval $(0, \pi / 2)$. This contradiction establishes the theorem. Q.E.D.

In other words. Theorem 4.2.1 says $\bar{\eta}_{1}(\alpha)$ exists iff no principal solution $(x, y)_{\theta}$ satisfies $x_{\theta}(t)>0$ and $y_{\theta}(t)>0$ on $(\alpha, \infty)$.

4.2.2. THEOREM. $\bar{\mu}_{1}(\alpha)$ exists iff for all $\theta \in(0, \pi / 2),(x, y)_{\theta} \in I$ II U I IV.

4.3. THEOREM. $\quad \bar{\eta}_{1}(\alpha)$ exists iff $\bar{\mu}_{1}(\alpha)$ exists. Moreover, $\bar{\mu}_{1}(\alpha)<\bar{\eta}_{1}(\alpha)$.

Proof. The first part is just a corollary of Theorems 4.2.1 and 4.2.2. Now let $\bar{\eta}_{1}(\alpha), \bar{H}_{1}(\alpha)$ be attained by principal solutions $(x, y)_{\phi},(x, y)_{\psi}$ respectively, where $\psi \geqslant \phi$. $\bar{\mu}_{1}(\alpha)$ is certainly smaller than the first zero of $x_{\psi}$ which is in turn smaller than $\bar{\eta}_{1}(\alpha)$ by Theorem 3.5.1. Q.E.D.

Thus far necessary and sufficient conditions have been established for existence of $\bar{\eta}_{1}(\alpha)$ and $\bar{\mu}_{1}(\alpha)$. It remains to show that $\bar{\eta}_{1}(\alpha)$ and $\bar{\mu}_{1}(\alpha)$ are actually the systems-conjugate and systems-focal points for (1).

\subsection{THEOREM. $\bar{\eta}_{1}(\alpha)=\hat{\eta}_{1}(\alpha)$ and $\bar{\mu}_{1}(\alpha)=\hat{\mu}_{1}(\alpha)$.}

Proof. By definition $\hat{\eta}_{1}(\alpha) \leqslant \bar{\eta}_{1}(\alpha)$. Moreover by Lemma 2.3.1, any solution $(x, y)$ of $(1)$ with $(x, y)(\alpha)=0$ and $x^{\prime}(\alpha) \leqslant 0, y^{\prime}(\alpha) \geqslant 0$ remains in the second quadrant on $\left(\alpha, \bar{\eta}_{1}(\alpha)\right.$ ]. Hence it is sufficient to consider $\hat{\eta}_{1}(\alpha)$ and $\bar{\eta}_{1}(\alpha)$ being attained by the principal solutions $(x, y)_{\hat{\theta}}$ and $(x, y)_{\bar{\theta}}$ respectively, with $\hat{\theta}>\bar{\theta}$. Now $(x, y)=(x, y)_{\hat{\theta}}-(x, y)_{\bar{\theta}}$ is a solution of (3) such that $(x, y)(\alpha)=0$ and $x^{\prime}(\alpha) \leqslant 0, y^{\prime}(\alpha) \geqslant 0$. Again by Lemma 2.3.1, $y(t)>0$ on $\left(\alpha, \bar{\eta}_{1}(\alpha)\right]$ which in turn implies $y_{\hat{\theta}}(t)>0$ on $\left(\alpha, \bar{\eta}_{1}(\alpha)\right]$. The first part of the theorem is proved. The proof of the second part is similar. Q.E.D.

5. Comparison theorems. The claim that a force field possessing a conjugate point implies the existence of a smaller conjugate point for any stronger force field was made in the introduction. While it is not possible to prove this fact directly, an introduction of the "reciprocal" system for (1) facilitates a proof. For convenience, (1) is rewritten here, 


$$
\left(\begin{array}{l}
x \\
y
\end{array}\right)^{n}+\left(\begin{array}{ll}
a & b \\
c & d
\end{array}\right)\left(\begin{array}{l}
x \\
y
\end{array}\right)=0
$$

together with its reciprocal system

$$
\left(\begin{array}{l}
u \\
v
\end{array}\right)^{\prime \prime}+\left(\begin{array}{ll}
a & c \\
b & d
\end{array}\right)\left(\begin{array}{l}
u \\
v
\end{array}\right)=0
$$

5.1.1. THEOREM. Let $\hat{\eta}_{1}(\alpha), \hat{\xi}_{1}(\alpha)$ be the systems-conjugate points for (1) and (6) respectively. Then $\hat{\eta}_{1}(\alpha)$ exists iff $\hat{\xi}_{1}(\alpha)$ exists. Moreover $\hat{\eta}_{1}(\alpha)=$ $\hat{\xi}_{1}(\alpha)$.

Proof. By Theorem 4.2.1, $\hat{\xi}_{1}(\alpha)$ does not exist if and only if there is a principal solution $(u, v)_{\varphi}$ such that $u_{\varphi}(t)>0$ and $v_{\varphi}(t)>0$ on $(\alpha, \infty)$. Assume now $\hat{\eta}_{1}(\alpha)$ exists and is attained by $(x, y)_{\phi}$. Consider the identity

$$
\begin{gathered}
{\left[(u, v)_{\varphi}\left(\begin{array}{l}
x \\
y
\end{array}\right)_{\phi}^{\prime}-(u, v)_{\varphi}^{\prime}\left(\begin{array}{l}
x \\
y
\end{array}\right)_{\phi}\right]^{\prime}=(u, v)_{\varphi}\left(\begin{array}{l}
x \\
y
\end{array}\right)_{\phi}^{\prime \prime}-(u, v)_{\varphi}^{\prime \prime}\left(\begin{array}{l}
x \\
y
\end{array}\right)_{\phi}} \\
=(u, v)_{\varphi}\left(\begin{array}{l}
x \\
y
\end{array}\right)_{\phi}^{\prime \prime}+(u, v)_{\varphi}\left(\begin{array}{ll}
a & b \\
c & d
\end{array}\right)\left(\begin{array}{l}
x \\
y
\end{array}\right)_{\phi} \\
=(u, v)_{\varphi}\left[\left(\begin{array}{l}
x \\
y
\end{array}\right)_{\phi}^{\prime \prime}+\left(\begin{array}{ll}
a & b \\
c & d
\end{array}\right)\left(\begin{array}{l}
x \\
y
\end{array}\right)_{\phi}\right]=0 .
\end{gathered}
$$

However

$$
0=\left[(u, v)_{\varphi}\left(\begin{array}{l}
x \\
y
\end{array}\right)_{\phi}^{\prime}-(u, v)_{\varphi}^{\prime}\left(\begin{array}{l}
x \\
y
\end{array}\right)_{\phi}\right]_{\alpha}^{\hat{n}_{1}(\alpha)}=\left(u_{\varphi} x_{\phi}^{\prime}+v_{\varphi} y_{\phi}^{\prime}\right)\left(\hat{\eta}_{1}(\alpha)\right)<0
$$

is a contradiction. Hence if $\hat{\eta}_{1}(\alpha)$ exists, then $\hat{\xi}_{1}(\alpha)$ exists. The converse is also true by symmetry. Moreover, the same argument establishes $\hat{\eta}_{1}(\alpha)=\hat{\xi}_{1}(\alpha)$. Q.E.D.

It is interesting to note that the concept of "reciprocal" for second and fourth order selfadjoint differential equations has already been utilized [5], [6], [9] in studying the oscillatory behavior of their solutions. Theorem 5.1.1 serves yet another such example. The next theorem is proved by similar methods.

5.1.2. THEOREM. Let $\hat{\mu}_{1}(\alpha), \hat{\nu}_{1}(\alpha)$ be the systems-focal points for (1) and (6) respectively. Then $\hat{\mu}_{1}(\alpha)$ exists iff $\hat{\nu}_{1}(\alpha)$ exists. Moreover $\hat{\mu}_{1}(\alpha)=\hat{\nu}_{1}(\alpha)$.

The above properties of the reciprocal system enables us to establish the following

5.2.1. THEOREM. Let $\hat{\sigma}_{1}(\alpha)$ be the systems-conjugate point for the following system 


$$
\left(\begin{array}{l}
X \\
Y
\end{array}\right)^{\prime \prime}+\left(\begin{array}{ll}
a & b \\
c & d
\end{array}\right)\left(\begin{array}{l}
X \\
Y
\end{array}\right)+\left(\begin{array}{ll}
a_{1} & b_{1} \\
c_{1} & d_{1}
\end{array}\right)\left(\begin{array}{l}
X \\
Y
\end{array}\right)=0
$$

where $a_{1}, b_{1}, c_{1}, d_{1}$ are nonnegative and continuous on $[\alpha, \infty)$. Let $\hat{\eta}_{1}(\alpha)$ be the systems-conjugate point for (1). If $\hat{\eta}_{1}(\alpha)$ exists, then $\hat{\sigma}_{1}(\alpha)$ exists. Moreover, $\hat{\sigma}_{1}(\alpha) \leqslant \hat{\eta}_{1}(\alpha)$.

Proof. It is equivalent to prove the theorem for $\hat{\xi}_{1}(\alpha)$ where $\hat{\xi}_{1}(\alpha)=$ $\hat{\eta}_{1}(\alpha)$ is the systems-conjugate point for (6). Assume to the contrary that $\hat{\sigma}_{1}(\alpha)$ does not exist. Thus there exists $(X, Y)_{\phi}$ such that $X_{\varphi}(t)>0, Y_{\varphi}(t)>0$ on $(\alpha, \infty)$. Let $\hat{\xi}_{1}(\alpha)$ be attained by the principal solution $(u, v)_{\varphi}$ and consider the identity

$$
\begin{array}{r}
{\left[(u, v)_{\varphi}\left(\begin{array}{l}
X \\
Y
\end{array}\right)_{\phi}^{\prime}-(u, v)_{\varphi}^{\prime}\left(\begin{array}{l}
X \\
Y
\end{array}\right)_{\phi}\right]^{\prime}=(u, v)_{\varphi}\left(\begin{array}{l}
X \\
Y
\end{array}\right)_{\phi}^{n}-(u, v)_{\varphi}^{\prime \prime}\left(\begin{array}{l}
X \\
Y
\end{array}\right)_{\phi}} \\
=(u, v)_{\varphi}\left(\begin{array}{l}
X \\
Y
\end{array}\right)_{\phi}^{\prime \prime}+(u, v)_{\varphi}\left(\begin{array}{ll}
a & b \\
c & d
\end{array}\right)\left(\begin{array}{l}
X \\
Y
\end{array}\right)_{\phi} \\
=u_{\varphi}\left(X_{\phi}^{\prime \prime}+a X_{\phi}+b Y_{\phi}\right)+v_{\varphi}\left(Y_{\phi}^{\prime \prime}+c X_{\phi}+d Y_{\phi}\right) .
\end{array}
$$

Now since $X_{\phi}(t)>0$ and $Y_{\phi}(t)>0$ on $(\alpha, \infty)$, (7) implies $X_{\phi}^{\prime \prime}+a X_{\phi}+b Y_{\phi}<$ 0 and $Y_{\phi}^{\prime \prime}+d Y_{\phi}+c Y_{\phi}<0$. Thus the identity reduces to an inequality:

$$
\left[(u, v)_{\varphi}\left(\begin{array}{l}
X \\
Y
\end{array}\right)_{\phi}^{\prime}-(u, v)_{\varphi}^{\prime}\left(\begin{array}{l}
X \\
Y
\end{array}\right)_{\phi}\right]^{\prime} \leqslant 0 \text { on }\left[\alpha, \hat{\xi}_{1}(\alpha)\right] .
$$

But

$$
0>\left[(u, v)_{\varphi}\left(\begin{array}{l}
X \\
Y
\end{array}\right)_{\phi}^{\prime}-(u, v)_{\varphi}^{\prime}\left(\begin{array}{l}
X \\
Y
\end{array}\right)_{\phi}\right]_{\alpha}^{\hat{\xi}_{1}(\alpha)}=-\left(u_{\varphi}^{\prime} X_{\phi}+v_{\varphi}^{\prime} Y_{\phi}\right)\left(\hat{\xi}_{1}(\alpha)\right) \geqslant 0
$$

is absurd. Similar arguments establish $\hat{\sigma}_{1}(\alpha) \leqslant \hat{\xi}_{1}(\alpha)$. Q.E.D.

5.2.2. THEOREM . Let $\hat{\mu}_{1}(\alpha)$ and $\hat{\kappa}_{1}(\alpha)$ be the systems-focal points for (1) and (7) respectively. If $\hat{\mu}_{1}(\alpha)$ exists, then $\hat{\kappa}_{1}(\alpha)$ also exists. Moreover, $\hat{\kappa}_{1}(\alpha)$ $<\hat{\mu}_{1}(\alpha)$.

Using the same technique as above, the following is easily established.

5.3. THEOREM. If $\hat{\eta}_{1}(\alpha)$ does not exist then for all $\beta \in(\alpha, \infty), \hat{\eta}_{1}(\beta)$ does not exist also.

Proof. Assume to the contrary that $\hat{\eta}_{1}(\beta)$ exists and let $\hat{\xi}_{1}(\beta)$ be the corresponding systems-conjugate point for the reciprocal system (6) achieved by $(u, v)_{\varphi}$. Then there is a principal solution $(x, y)$ for $(1)$ such that $x_{\phi}>0$, 
$y_{\phi}>0$ on $(\alpha, \infty)$ and as in the proof of Theorem 5.1.1.,

$$
0=(u, v)_{\varphi}\left(\begin{array}{l}
x \\
y
\end{array}\right)_{\phi}^{\prime}-\left.(u, v)_{\varphi}^{\prime}\left(\begin{array}{l}
x \\
y
\end{array}\right)_{\phi}\right|_{\beta} ^{\hat{\xi}_{1}(\beta)}>0
$$

which is absurd. Q.E.D.

6. Necessary and sufficient conditions for existence of $\hat{\eta}_{1}(\alpha)$. We say equation (1) is systems-conjugate in $(\alpha, \beta)$ (or $(\alpha, \beta])$ if $\hat{\eta}_{1}(\alpha)$ exists and $\hat{\eta}_{1}(\alpha)$ $<\beta$ (or $\leqslant \beta$ ). Otherwise (1) is said to be systems-disconjugate in $(\alpha, \beta)$ (or $(\alpha$, $\beta]$ ). In this section, a number of conjugacy criteria for (1) will be derived. There are at least two types of criteria. The first is based on Theorem 4.2.1, while the second is derived from the comparison theorem.

6.1. TheOREM. If either $u^{\prime \prime}+a(t) u=0$ or $v^{\prime \prime}+d(t) v=0$ is oscillatory on $(\alpha, \infty)$, then $(1)$ is systems-conjugate in $(\beta, \infty)$ for every $\beta>\alpha$.

ProOF. By Lemma 2.2, if either $u^{\prime \prime}+a u=0$ or $v^{\prime \prime}+d v=0$ is oscillatory on $(\alpha, \infty)$ implies nonexistence of solution $(x, y)$ of (1) satisfying $x(t)>0$ and $y(t)>0$ on $(\beta, \infty)$ for every $\beta>\alpha$. Hence equation (3) is systems-conjugate by Theorem 4.2.1. Q.E.D.

6.2. THEOREM. If either $\int^{\infty} b(t) d t$ or $\int^{\infty} c(t) d t$ diverges, then (1) is systems-conjugate in $(\beta, \infty)$ for every $\beta>\alpha$.

Proof. Assume to the contrary that there is a principal solution $(x, y)_{\varphi}$ such that $x_{\varphi}(t)>0$ and $y_{\varphi}(t)>0$ on $(\beta, \infty)$, then by Lemma $2.1, x_{\varphi}^{\prime}>0, y_{\varphi}^{\prime}>$ 0 on $(\beta, \infty)$. Now

$$
0<x_{\varphi}^{\prime}(t)=x_{\varphi}^{\prime}(\gamma)+\int_{\gamma}^{t}-a x_{\varphi}-b y_{\varphi} \leqslant x_{\varphi}^{\prime}(\gamma)-\int_{\gamma}^{t} b y_{\varphi} \leqslant x_{\varphi}^{\prime}(\gamma)-y_{\varphi}(\gamma) \int_{\gamma}^{t} b
$$

where $\gamma>\beta$. Hence if $\int^{\infty} b=\infty$, then the right-hand side of the inequality diverges to $-\infty$ which is absurd. Q.E.D.

6.3. CoROllary. If $a=1 / d \neq 0$ or $b=1 / c$ then (1) is systems-conjugate in $(\beta, \infty)$ for every $\beta>\alpha$.

6.4. THEOREM. Let $a=d$ and $b=c$ in equation (1). Then (1) is systems-disconjugate on $(\alpha, \beta)$ if and only if the second order equation

$$
u^{\prime \prime}+(a+b) u=0
$$

is disconjugate on $(\alpha, \beta)$.

ProOF. Let $u$ be the solution of (6) satisfying $u(\alpha)=0$ and $u^{\prime}(\alpha)=$ $1 / \sqrt{2}$. Then $(x, y)_{\pi / 4} \equiv(u, u)$ is easily shown to be a principal solution of (1). 
Now if $(8)$ is disconjugate on $(\alpha, \beta)$, then $\hat{\eta}_{1}(\alpha)$ cannot exist in $(\alpha, \beta)$ by Theorem 3.5.1. Conversely, if $(8)$ is not disconjugate on $(\alpha, \beta)$, Theorem 3.5.1 again implies that $\hat{\eta}_{1}(\alpha)$ is less than or equal to the first zero of $u$. Q.E.D.

6.5. THEOREM. Let $a=d$. If

$$
u^{\prime \prime}+(a+(b+c) / 2) u=0
$$

is disconjugate on $(\alpha, \beta)$, then (1) is systems-disconjugate on $(\alpha, \beta)$.

Proof. According to Hartman and Wintner [5], if the following "symmetrized" system of (3)

$$
\left(\begin{array}{l}
x \\
y
\end{array}\right)^{\prime \prime}+\left(\begin{array}{cc}
a & (b+c) / 2 \\
(b+c) / 2 & d
\end{array}\right)\left(\begin{array}{l}
x \\
y
\end{array}\right)=0
$$

is systems-disconjugate, then (1) is also systems-disconjugate. Now since $a=d$ it follows from Theorem 6.4 that disconjugacy of (9) implies systems-disconjugacy of (10) which in turn implies systems-disconjugacy of (1). Q.E.D.

6.6. THEOREM. If equation (1) is systems-disconjugate on $(\alpha, \infty)$, then

$$
u^{\prime \prime}+(\min (a, d)+\min (b, c)) u=0
$$

is disconjugate on $(\alpha, \infty)$.

Proof. By the comparison theorem, systems-disconjugacy of (1) implies systems-disconjugacy of

$$
\left(\begin{array}{l}
x \\
y
\end{array}\right)^{\prime \prime}+\left(\begin{array}{cc}
\min (a, d) & \min (b, c) \\
\min (b, c) & \min (a, d)
\end{array}\right)\left(\begin{array}{l}
x \\
y
\end{array}\right)=0
$$

which in turn implies disconjugacy of (11) by Theorem 6.5. Q.E.D.

6.7. ThEOREM. If $(1)$ is systems-conjugate in $(\alpha, \infty)$ then

$$
u^{\prime \prime}+(\max (a, d)+(b+c) / 2) u=0, \quad u(\alpha)=0
$$

is not disconjugate on $(\alpha, \infty)$.

PROOF. This follows from the comparison theorem and Theorem 6.5. Q.E.D.

7. Application of fourth order equations. It is shown in [2] that as far as oscillation properties are concerned, (4) can be reduced to the form

$$
l[u] \equiv\left(p_{2}(t) u^{\prime \prime}\right)^{\prime \prime}-\left(p_{1}(t) u^{\prime}\right)^{\prime}+q(t) u+p_{0}(t) u=0 .
$$

It is also shown that with minor restriction on the coefficients there is a one-to- 
one correspondence between equations of form (1) and (12) defined by the following set of transformations:

$$
\begin{gathered}
x(t) \equiv u(t), \quad y(t)=-\frac{1}{b(t)} x^{\prime \prime}(t)-\frac{a(t)}{b(t)} x(t), \\
a=-\frac{p_{1}-\int_{\alpha}^{t} q}{2 p_{2}}, \quad b=-\frac{1}{p_{2}}>0, \\
c=p_{0}-\frac{p_{1}^{\prime \prime}-q^{\prime}}{2}-\frac{p_{1}^{2}-\left(\int_{\alpha}^{t} q\right)^{2}}{4 p_{2}}, \quad d=-\frac{p_{1}+\int_{\alpha}^{t} q}{2 p_{2}} .
\end{gathered}
$$

It becomes routine then to apply the results for (1) to equation (12). However, it is of interest to examine the hypotheses of $a, b, c, d$ and the meaning of $\hat{r}_{1}(\alpha)\left(\hat{\mu}_{1}(\alpha)\right)$ in terms of (12). Now $b>0$ implies $p_{2}<0$, while $a, d \geqslant$ 0 require $\int_{\alpha}^{t} q \leqslant-p_{1}$. Thus the nonnegativeness of $a$ and $d$ restrict the growth of the "nonselfadjoint" term of $q(t)$. The boundary condition $(x, y)(\alpha)=0=$ $(x, y)(\beta)$ implies $u(\alpha)=u^{\prime \prime}(\alpha)=0=u(\beta)=u^{\prime \prime}(\beta)$, while $(x, y)(\alpha)=0=$ $(x, y)^{\prime}(\beta)$ requires $u(\alpha)=u^{\prime \prime}(\alpha)=0=u^{\prime}(\beta)=\left[p_{2} x^{\prime \prime}+\left(\int_{\alpha}^{t} q-p_{1}\right) x / 2\right]^{\prime}(\beta)$. Hence, Theorem 4.4 says that the smallest $\beta \in(\alpha, \infty)$ such that $u(\alpha)=u^{\prime \prime}(\alpha)=$ $0=u(\beta)=u^{\prime \prime}(\beta)$ is realized by a positive convex (downward) function. Such a function exists if conditions corresponding to those of $\S 6$ are satisfied by $p_{2}$, $p_{1}, p_{0}$, and $q$.

The author wishes to sincerely thank Professor K. Kreith for his invaluable advice and assistance in this paper.

\section{REFERENCES}

1. W. M. Whyburn, On self-adjoint ordinary differential equations of the fourth order, Amer. J. Math. 52 (1930), 171-196.

2. K. Kreith, A nonselfadjoint dynamical system, Proc. Edinburgh Math. Soc. (2) 19 (1974/75), 77-87. MR 49 \# 7513.

3. J. H. Barrett, Systems-disconjugacy of a fourth-order differential equation, Proc. Amer. Math. Soc. 12 (1961), 205-213. MR 24 \# A304.

4. G. A. Bliss and I. J. Schoenberg, On separation, comparison, and oscillation theorems for self-adjoint systems of linear second-order differential equations, Amer. J. Math. 53 (1931), $781-800$.

5. P. Hartman and A. Wintner, On disconjugate differential systems, Canad. J. Math. 8 (1956), 72-81. MR 17, 611.

6. W. Leighton and Z. Nehari, On the oscillation of solutions of self-adjoint linear differential equations of the fourth order, Trans. Amer. Math. Soc. 89 (1958), 325-377. MR 21 \# 1429.

7. W. Leighton, Some elementary Sturm theory, J. Differential Equations 4 (1968), 187-193; Addendum, ibid. 6 (1969), 196. MR 37 \# 506; 39 \# 3084.

\section{DEPARTMENT OF MATHEMATICS, UNIVERSITY OF CALIFORNIA, DAVIS, CALIFORNIA 95616}

Current address: Department of Mathematics, University of British Columbia, Vancouver, B. C., Canada V6T 1W5 